\title{
Factores críticos en la gestión de conocimiento en países: un análisis global del proceso
}

\author{
María OBESO \\ Universidad de Cantabria \\ obesom@unican.es \\ María SARABIA \\ Universidad de Cantabria \\ sarabiam@unican.es \\ José María SARABIA \\ Universidad de Cantabria \\ sarabiaj@unican.es
}

Recibido: 25-02-2012

Aceptado: 01-09-2012

\section{RESUMEN}

En la sociedad actual, el conocimiento se ha convertido en un activo muy valioso fuente de ventajas competitivas. Sin embargo aunque en el caso de las empresas esta importancia ha sido muy analizada en las últimas décadas, no se han realizado hasta el momento grandes esfuerzos en el análisis de la gestión del conocimiento en países, lo que ha motivado el presente estudio donde identificamos los factores críticos de este proceso en países a partir de un análisis factorial exploratorio: (1) Potencial de creación de conocimiento, (2) Productividad TIC, (3) Internacionalización del conocimiento, (4) Resultados de investigación y (5) Autopista de la educación. Posteriormente se propone un análisis comparativo de las principales variables relacionadas con la inversión y el resultado de conocimiento que permite conocer cómo los principales grupos de países del mundo (G-20, Unión Europea y APEC, entre otros) gestionan este activo.

Palabras clave: Gestión del conocimiento, Estrategia de países, Análisis factorial, G-20, Unión Europea, OCDE, Organizaciones 


\title{
Critical factors in knowledge management in countries: A global analysis about the process
}

\begin{abstract}
At present society, knowledge is a fundamental asset in order to obtain competitive advantages. However, although this asset is considered as essential in enterprises, there are not some studies about knowledge management in countries. In this scenario, we propose an interesting analysis about how countries manage their knowledge identifying five critical factors in this process: (1) Knowledge creation potential, (2) ICT Productivity, (3) Knowledge internationalization, (4) Research results and (5) Education motorway. After we propose a comparative analysis between countries using variables linked to knowledge investment and result, with the aim of know how the principal groups of countries (G-20, European Union and APEC, between others) manage their assets.
\end{abstract}

Keywords: Knowledge management, Countries strategy, Factor analysis, G-20, European Union, ODCE, Organizations

Sumario: 1. Introducción. 2. Metodología. 3. Resultados. 4. Conclusiones. Bibliografía.

\section{INTRODUCCIÓN}

En la sociedad actual el conocimiento se ha convertido en un activo muy valioso, tanto para las organizaciones en general como para las personas en particular. En los últimos años han proliferado las investigaciones relacionadas con este tema (Kebede 2010), y su popularidad es tal que incluso es conocida como sociedad del conocimiento (Sakaiya 1991). Este nuevo concepto de sociedad ha surgido para dar respuesta al cambio que se ha producido en el mundo, pasando de una sociedad industrial a una sociedad donde la producción del conocimiento y su uso se presentan como pilar básico de sus actividades (Drucker 1993; Kakabadse et al. 2001; Morrow 2001; Oluic-Vukovic 2001; Passerini 2007; Kebede 2010).

Sin embargo, a pesar de este reconocimiento de la importancia del conocimiento en la sociedad actual y del incremento de publicaciones relacionadas con este tema como valor fundamental para el desempeño de las empresas (Villela y Muñiz 2010), no han sido muchas las investigaciones sobre el conocimiento en los países. Los países también deben de gestionar el conocimiento, presente tanto en la mente de los individuos que lo conforman (Sharratt y Usoro 2003) como en grandes y pequeñas empresas (Fletcher y Polychronakis 2007), para lograr ser competitivos a nivel mundial.

En este escenario, esta investigación supone dar respuesta a este gap existente en la teoría de la gestión del conocimiento en las organizaciones ofreciendo un análisis factorial exploratorio que identifica los factores críticos a analizar por aquellos países que deseen incrementar su competitividad y desempeño a través del conocimiento. Además este análisis no solo contribuye a la teoría, sino que también 
sirve de soporte para la toma de decisiones de todos aquellos gobiernos interesados en este proceso.

La estructura del artículo es la siguiente: en el siguiente apartado se ofrece el marco teórico de la investigación mediante una revisión de las contribuciones realizadas en el campo de la gestión del conocimiento en los países, detallando al finalizar el epígrafe el objetivo del trabajo que se ha adelantado en esta introducción. La siguiente sección recoge la metodología utilizada, justificando las técnicas estadísticas, la base de datos utilizada y las variables seleccionadas. Posteriormente se exponen los resultados del análisis estadístico y se ofrece la discusión y las aportaciones que se realizan. Finalmente la conclusión recoge las principales contribuciones de esta investigación, las implicaciones prácticas que conlleva, las limitaciones que se ofrecen y, finalmente, las futuras líneas que se derivan del trabajo.

La gestión del conocimiento es una actividad que se ha venido realizando desde la antigüedad, cuando padres, artesanos, soldados o académicos han transmitido su experiencia y el secreto de su éxito a hijos y aprendices (Obeso et al. 2012). Esto es debido fundamentalmente al carácter personal del mismo que relaciona directamente la práctica de esta gestión con las personas que forman parte de las organizaciones (Drucker 1991; Zhou et al. 2010).

En relación a esto, en una sociedad como la actual donde la globalización ha fomentado la competencia de las organizaciones, la gestión del conocimiento se ha identificado como herramienta fundamental (Macintosh 1996; Jimes y Lucardie 2003). Sin embargo, como ya se había adelantado en la introducción, mientras que en el caso de las empresas las investigaciones en este campo se han incrementado notablemente, la gestión del conocimiento en los países no es reconocida aún como estrategia clave para la competitividad internacional y las contribuciones en esta área han sido escasas.

Adams realizó un estudio en 1990 en el cual analizó la industria manufacturera de los Estados Unidos de América (EEUU) en el período 1953-1980 y encontró evidencias de que el conocimiento técnico ha contribuido significativamente al crecimiento de la productividad total de esta industria. Siguiendo esta línea Poole y Bernard (1992) proporcionan evidencia de la relación entre la innovación y el crecimiento de la productividad durante el período 1961-1985 utilizando las innovaciones militares en el campo militar en Canadá (Chen y Dahlman 2004).

Años más tarde, Jones (2002) determinó que el crecimiento de un país se encuentra estrechamente relacionado con las nuevas ideas generadas en el mundo. Este stock de ideas es proporcional al esfuerzo en investigación de todo el mundo, que a su vez es proporcional a la población total de los países. En base a esto el capital humano es fundamental para obtener resultados de investigación y fomentar el crecimiento. En este escenario, esta investigación pretende dar respuesta a cómo los países gestionan su conocimiento. 
En relación a esto, Lederman y Maloney (2003), utilizando regresiones con paneles de datos del promedio de cinco años entre 1975 a 2000 en más de 53 países, concluyeron que un incremento de un punto porcentual en la proporción del gasto total en investigación se traduce en un aumento de la tasa de crecimiento del PIB en 0,78 puntos porcentuales. Por su parte, Guellec y Van Pottelsberghe (2001) sostienen que la inversión en investigación y desarrollo tiene efectos positivos en el crecimiento de la productividad.

Recientemente se han publicado varios artículos en revistas de impacto relativos a este tema. Por ejemplo Martinus (2010), relaciona aspectos de una región como la conectividad, la educación y capacitación, la creatividad y la cultura, el agrupamiento industrial y la diversidad como fundamentales para crear un entorno socioeconómico donde se desarrolle la innovación y el conocimiento. Siguiendo a esta autora, "en una economía del conocimiento, la planificación urbana debe de aprovechar la energía humana a través de la infraestructura para ampliar el desarrollo social, la formación de capital, la transferencia de conocimiento y, por lo tanto, incrementar la productividad basada en el desarrollo de conocimiento de una sociedad" (Martinus 2010,. 738).

Por otro lado Sarabia et al. (2012) identifican una estrecha relación entre gestión del conocimiento en países y desempeño a través de un análisis de esta gestión en la Unión Europea. Estos autores destacan la existencia en esta región de un conjunto de países denominado Países del conocimiento que se caracterizan por elevadas inversiones en variables relacionadas con este activo intangible y buenos resultados tanto relativos al conocimiento como generales de los países.

En este escenario, el objetivo principal de este trabajo es profundizar en los factores críticos relacionados con la gestión de conocimiento en los países de todo el mundo, contribuyendo de esta forma en la toma de decisiones relativa a la estrategia de los gobiernos.

Además se propone otro objetivo relacionado estrechamente con el anterior: identificar similitudes y diferencias de comportamientos relacionados con el conocimiento en distintos grupos de países que serán analizados: Unión Europea, Organización para la Cooperación y el Desarrollo Económico (OCDE), G-20, CAIRNS, Foro de Cooperación Económica Asia-Pacífico (APEC), MERCOSUR, BRIC y NEXT-11 (N-11). Este segundo objetivo permite conocer qué grupos de países están gestionando mejor su conocimiento de forma que se orienta a todos aquellos gobiernos interesados en las actuaciones que pueden llevar a cabo para mejorar su desempeño a través de este activo intangible. 


\section{METODOLOGÍA}

Los datos de este análisis han sido obtenidos de la base de datos de libre acceso publicada por el Banco Mundial (2010). El estudio se ha hecho con datos del 2005, año cuya información sobre los países tiene menos valores perdidos.

El nivel del análisis es mundial, y para la realización del mismo se ha seleccionado una muestra de países de todo el mundo en función de su pertenencia a organizaciones de reconocimiento internacional: Unión Europea, OCDE, G-20, CAIRNS, APEC, MERCOSUR, BRIC y NEXT-11. Como resultado de esta muestra, se incluyen 67 países que representan todos los continentes. La selección de las organizaciones se ha hecho en base a criterios de localización, de importancia de los grupos en la actualidad y de la importancia creciente de los mismos. Con respecto al criterio de localización se han seleccionado las siguientes organizaciones: MERCOSUR, APEC y Unión Europea. En cuanto a la importancia actual, tanto a nivel económico como político, de las mismas, la elección ha sido de: OCDE, G-20 y Unión Europea. Por su parte los países BRIC y los N-11 han sido seleccionados por su importancia creciente en la sociedad actual. Finalmente, y con el objetivo de que la muestra sea representativa, se ha seleccionado la organización de países CAIRNS como referentes en el sector primario. Los 67 países seleccionados ocupan el 64,57 por ciento de la superficie de la tierra y en ellos vive el aproximadamente el 80 por ciento de la población de todo el mundo. Estas cifras justifican la elección de la muestra.

Se han seleccionado para el análisis 16 variables relacionadas directa o indirectamente con la gestión del conocimiento en países (ver Tabla 1) y que previamente han sido destacadas en las contribuciones realizadas por diversos autores como importantes en esta área (ver Tabla 2). Estas variables pueden ser clasificadas en las siguientes categorías:

(1) Creación de conocimiento: se incluyen todas aquellas variables relacionadas con la creación de nuevo conocimiento en las economías.

(2) Transferencia de conocimiento: se incluyen variables relacionadas con la transferencia de conocimiento dentro de una sociedad.

(3) Desempeño general: se incluyen variables relacionadas con el resultado general de los países, medido a través del PIB.

En esta investigación se aplica la técnica de análisis factorial exploratorio descrita por Kim y Mueller $(1994,1)$ como una "técnica estadística cuyo objetivo es representar un grupo de variables en términos de un número reducido de factores subyacentes". En este sentido, el análisis factorial es comúnmente utilizado para "identificar los factores subyacentes o las dimensiones que reflejan una variabilidad común" (Hair et al 1995, 375). Esta técnica ayuda a los investigadores 
a "dotar de sentido a un elevado número de datos interrelacionados" (Hair et al. 1995, 404). El análisis factorial es utilizado, por lo tanto, para identificar los factores críticos a la hora de gestionar el conocimiento en los países.

Se ha utilizado el método de análisis de componentes principales (ACP) rotando los factores mediante el método Varimax. Hair et al. $(1995,380)$ recomiendan la rotación porque "simplifica la estructura del factor y los resultados obtenidos son más significativos".

Tabla 1. Tipología de las variables utilizadas para el análisis

\begin{tabular}{|c|c|c|}
\hline Variables de descripción & Variables de inversión & Variables de resultado \\
\hline Densidad de población & Gasto TIC & Crecimiento PIB \\
$\mathrm{N}^{\circ}$ Investigadores & Gasto I+D & PIB per cápita \\
$\mathrm{N}^{\circ}$ Técnicos & Gasto Educación & Patentes \\
Usuarios Internet & & Artículos científicos \\
Pago regalías & Ratio nuevas empresas \\
Cobro regalías & \\
Exportaciones alta tecnología & & \\
Exportaciones TIC & & \\
Importaciones TIC & & \\
\hline
\end{tabular}

Fuente: Elaboración propia

Tabla 2. Variables seleccionadas y autores que resaltan su importancia

\begin{tabular}{|c|c|}
\hline Variables & Autores que destacan su importancia \\
\hline Densidad de población & $\begin{array}{l}\text { Acs, Anselin y Vorga, 2002; Jones, 2002; Li, 2002; Varga y Schalk, 2004; Arnold, } \\
\text { 2006; Berliant, Reed y Wang, 2006; Tsé, 2008; Martinus, } 2010 .\end{array}$ \\
\hline $\begin{array}{l}\text { Número de usuarios de } \\
\text { Internet }\end{array}$ & Chen y Dahlman, 2004. \\
\hline $\begin{array}{l}\text { Número de } \\
\text { investigadores }\end{array}$ & $\begin{array}{l}\text { OECD, 2001; Florida, 2002; Jones, 2002; López-Bassols, 2002; Glaeser y Saiz, 2003; } \\
\text { Chen y Dahlman, 2004; Florida y Tinagli, 2004; Raspe y Van Oort, 2006; Lee y } \\
\text { Choi, 2008; Martinus, 2010. }\end{array}$ \\
\hline Número de técnicos & $\begin{array}{l}\text { OECD, 2001; Florida, 2002; Jones, 2002; López-Bassols, 2002; Glaeser y Saiz, 2003; } \\
\text { Chen y Dahlman, 2004; Florida y Tinagli, 2004; Raspe y Van Oort, 2006; Lee y } \\
\text { Choi, 2008; Martinus, } 2010 .\end{array}$ \\
\hline $\begin{array}{l}\text { Exportaciones de alta } \\
\text { tecnología }\end{array}$ & Cerquera y Klein, 2008; Polder, Leeuwen, Mohnen y Raymond, 2009. \\
\hline
\end{tabular}




\begin{tabular}{|c|c|}
\hline $\begin{array}{l}\text { Exportaciones e } \\
\text { importaciones de bienes } \\
\text { y servicios TIC }\end{array}$ & Cerquera y Klein, 2008; Polder, Leeuwen, Mohnen y Raymond, 2009. \\
\hline $\begin{array}{l}\text { Pagos y cobros de } \\
\text { regalías y tarifas de } \\
\text { licencia }\end{array}$ & Lee y Choi, 2008. \\
\hline Gasto en educación & $\begin{array}{l}\text { Chen y Dahlman, 2004; Goddard, 2007; Martínez-Fernández, Rerceretnam y Sharpe, } \\
2007 .\end{array}$ \\
\hline $\begin{array}{l}\text { Gasto en tecnologías de } \\
\text { la información y la } \\
\text { comunicación (TIC) }\end{array}$ & $\begin{array}{l}\text { Greenan, Topiol-Bensaid y Mairesse, 2001; Bresnahan et al, 2002; Cerquera y Klein, } \\
\text { 2008; Castiglione, 2009; Polder, Leeuwen, Mohnen y Raymond, } 2009 .\end{array}$ \\
\hline Gasto I+D & $\begin{array}{l}\text { Kogut y Zander, 1992; Henderson y Cockburn, 1994; Davidsson y Segerstrom, 1998; } \\
\text { Fleming, 2001; Guellec y van Pottelsberghe, 2001; Lederman y Maloney, 2003; Chen } \\
\text { y Dahlman, 2004; Lindström y Heshmati, 2005; Comisión Europea, } 2007 .\end{array}$ \\
\hline Crecimiento del PIB & $\begin{array}{l}\text { Guellec y van Pottelsberghe, 2001; Acs, Anselin y Varga, 2002; Jones, 2002; Li, } \\
\text { 2002; Lederman y Maloney, 2003; Chen y Dahlman, 2004; Varga y Schalk, 2004; } \\
\text { Arnold, 2006; Berliant, Reed y Wang, } 2006 .\end{array}$ \\
\hline PIB per cápita & $\begin{array}{l}\text { Guellec y van Pottelsberghe, 2001; Acs, Anselin y Varga, 2002; Jones, 2002; Li, } \\
\text { 2002; Lederman y Maloney, 2003; Chen y Dahlman, 2004; Varga y Schalk, 2004; } \\
\text { Arnold, 2006; Berliant, Reed y Wang, } 2006 .\end{array}$ \\
\hline Tasa de desempleo & $\begin{array}{l}\text { Guellec y van Pottelsberghe, 2001; Acs, Anselin y Varga, 2002; Jones, 2002; Li, } \\
\text { 2002; Lederman y Maloney, 2003; Chen y Dahlman, 2004; Varga y Schalk, 2004; } \\
\text { Arnold, 2006; Berliant, Reed y Wang, } 2006 .\end{array}$ \\
\hline Número de patentes & $\begin{array}{l}\text { Adams, 1991; Romer, 1990; North, 1991; Jones, 2002; Gans, Murray y Stern, 2005; } \\
\text { Raspe y Van Oort, 2006; Murray y O'Mahony, 2007; Furman y Stern, 2008; Lee y } \\
\text { Choi, } 2008 .\end{array}$ \\
\hline $\begin{array}{l}\text { Número de artículos } \\
\text { científicos }\end{array}$ & $\begin{array}{l}\text { Adams, 1991; Romer, 1990; North, 1991; Jones, 2002; Gans, Murray y Stern, 2005; } \\
\text { Raspe y Van Oort, 2006; Murray y O'Mahony, 2007; Furman y Stern, 2008; Lee y } \\
\text { Choi, } 2008 .\end{array}$ \\
\hline $\begin{array}{l}\text { Ratio de nuevas } \\
\text { empresas }\end{array}$ & $\begin{array}{l}\text { Hansen, Nohra y Tierney, 1999; Fletcher y Polychronakis, 2007; King, Kruger y } \\
\text { Pretorius, } 2007 \text {. }\end{array}$ \\
\hline
\end{tabular}

Fuente: Elaboración propia 


\section{RESULTADOS}

En esta sección se explican los resultados obtenidos en el análisis que dan lugar a las aportaciones originales de la investigación. En primer lugar, se identifican los parámetros de éxito en la gestión del conocimiento en los países, permitiendo conocer los aspectos fundamentales de la misma y, por tanto, centrar esfuerzos. En segundo lugar realizamos una comparación de las agrupaciones de países para conocer la situación de cada una de ellas.

A la vista de la adecuación del análisis factorial con estos datos y utilizando la matriz de componentes rotados (ver Tabla 3), cinco factores que explican más del 70 por ciento de la variabilidad de los datos (ver Tabla 4) son definidos Hair et al. $(1995,378)$ argumentan que "es común considerar una solución que represente el 60 por ciento de la variabilidad como satisfactoria", por lo que de nuevo se confirma la adecuación del análisis factorial. Los cinco factores identificados son los siguientes: (1) Potencial de creación de conocimiento, (2) Productividad TIC, (3) Resultados de investigación, (4) Internacionalización del conocimiento, y (5) Autopista de la educación.

Factor 1: Potencial de creación de conocimiento. Este primer factor representa el 29,76 por ciento de la variabilidad y recoge las siguientes variables: PIB per cápita, número de técnicos, cobro de regalías y tarifas de licencia, número de investigadores, número de usuarios de Internet por cada 100 habitantes, gasto en $\mathrm{I}+\mathrm{D}$, número de artículos científicos y crecimiento del PIB. Incluye por lo tanto, varias variables estrechamente relacionadas con el conocimiento como la inversión en $\mathrm{I}+\mathrm{D}$, el número de técnicos, el número de investigadores y el cobro de regalías y tarifas de licencia. En este sentido está relacionado con los trabajadores del conocimiento, que a su vez influyen en la creación del mismo. Respecto a la inversión en $\mathrm{I}+\mathrm{D}$, el conocimiento amplía las posibilidades de las personas promoviendo la creatividad y la imaginación (Nussbaum 2000). Esta inversión, por su parte, juega un papel fundamental en el crecimiento económico de los países, ya que es la base para la producción de conocimiento científico (Romer 1990b). Los artículos científicos publicados sirven de base para nuevo conocimiento que puede tomar forma de patente (Gans et al 2005). 
Tabla 3. Matriz de componentes rotados ${ }^{1}$

\begin{tabular}{|c|c|c|c|c|c|}
\hline & \multicolumn{5}{|c|}{ Componente } \\
\hline & 1 & 2 & 3 & 4 & 5 \\
\hline PIB per cápita & ,920 & - & 106 &, 117 & - \\
\hline Investigadores &, 885 &, 115 & - &,- 143 & 107 \\
\hline Usuarios de Internet &, 881 &, 227 &,- 165 & - & - \\
\hline Técnicos & 852 & - & - & 139 & - \\
\hline Artículos &, 823 & - & - &,- 152 & - \\
\hline Cobro de regalías & ,736 &, 113 & ,261 & - &,- 114 \\
\hline Gasto público I+D & ,716 & - & - &,- 430 & - \\
\hline Crecimiento PIB &,- 470 & - & 261 &, 238 &, 151 \\
\hline Exportaciones bienes TIC & - & ,941 & - & - & - \\
\hline Importaciones bienes TIC &,- 106 & ,930 &, 110 & - &, 115 \\
\hline Exportaciones alta tecnología & 180 &, 850 & 151 &,- 124 & - \\
\hline Gasto TIC & 186 &, 677 &,- 221 &,- 154 & - \\
\hline Densidad población & - &, 594 & - & ,315 &, 173 \\
\hline Exportación servicios TIC & - &,- 109 &, 864 & - & - \\
\hline Pago regalías & ,203 & ,301 & ,693 &, 119 & - \\
\hline Patentes & 327 &, 152 &,- 181 &,- 705 & 104 \\
\hline Ratio nuevas empresas & 423 &, 172 &,- 165 &, $\mathbf{5 7 0}$ & 267 \\
\hline Tasa desempleo &,- 242 &,- 115 &,- 137 & - &,- 776 \\
\hline Gasto educación &,- 313 &, 120 & - &, 130 &, 715 \\
\hline
\end{tabular}

Fuente: Elaboración propia

Tabla 4. Total de la varianza explicada

\begin{tabular}{|c|c|c|c|c|}
\hline \multirow[t]{2}{*}{ Componente } & \multicolumn{4}{|c|}{ Suma de las saturaciones al cuadrado de la rotación } \\
\hline & & Total & Porcentaje de la varianza & $\begin{array}{l}\text { Porcentaje } \\
\text { acumulado }\end{array}$ \\
\hline & 1 & 5,655 & 29,762 & 29,762 \\
\hline & 2 & 3,562 & 18,749 & 48,511 \\
\hline & 3 & 1,585 & 8,340 & 56,851 \\
\hline & 4 & 1,333 & 7,015 & 63,867 \\
\hline Factor & 5 & 1,326 & 6,980 & 70,846 \\
\hline
\end{tabular}

Fuente: Elaboración propia

Factor 2: Productividad TIC. Este segundo factor recoge aproximadamente el 18 por ciento de la variabilidad de los países e incluye variables relacionadas con la inversión en tecnologías de la información y la comunicación: exportaciones e importaciones de bienes relacionados con las tecnologías de la información y la comunicación, exportaciones de alta tecnología, gasto público en tecnología de la información y la comunicación y densidad de población. La inversión en tecnologías de la información y la comunicación propicia las exportaciones e importaciones relacionadas con la materia. A su vez, estas importaciones y exportaciones fomentan la participación y el intercambio de conocimiento con otros países, lo que se traduce

\footnotetext{
${ }^{1}$ Los valores menores de 0,1 han sido suprimidos de la tabla.
} 
en creación de conocimiento relacionada entre otros aspectos, con las exportaciones de alta tecnología. La inversión en TIC es comúnmente vista para procesar y organizar el trabajo dentro de la empresa (Greenan et al 2001). Un uso intenso de las TIC reduce el esfuerzo necesario en I+D (Cerquera y Klein 2008), lo que implica cierta complementariedad entre ambos aspectos (Polder et al. 2009). El conocimiento es personal y, por lo tanto, en la gestión del conocimiento es esencial centrarse en los individuos para extraerlo y que forme parte de la organización. La relación entre las TIC y la productividad de la empresa es en general, positiva (Bresnahan et al. 2002; Castiglione 2009).

Factor 3: Internacionalización del conocimiento. El tercer factor explica más del 8 por ciento de la variabilidad de los datos y en él se incluyen las variables siguientes: exportaciones de servicios TIC y pagos de regalías y tarifas de licencia. Está estrechamente relacionado con la exportación de conocimiento a otros países a través de contratos de licencia como pueden ser, por ejemplo, las franquicias.

Factor 4: Resultados de investigación. El factor 4 explica más del 7 por ciento de la variabilidad de los datos y recoge variables relacionadas con los resultados en la investigación: el ratio de nuevas empresas y el número de patentes solicitadas. Esto significa que este factor recoge los resultados de las diferentes estrategias que siguen los países para gestionar su conocimiento. Romer (1990b) especificó que la producción de conocimiento científico juega un papel fundamental en el crecimiento económico.

Factor 5: Autopista de la educación. El quinto y último factor identificado representa casi el 7 por ciento de la variabilidad de los países y destaca la importancia del gasto en educación, que se encuentra relacionado con el desempleo. La educación es fundamental para la existencia en el país de personas capaces de trabajar en áreas de alta y media tecnología, por lo que es vital que los gobiernos realicen inversiones en este campo. Por un lado la educación básica es indispensable para incrementar la capacidad de aprendizaje de las personas y el uso de la información que supondrá la base necesaria para profundizar posteriormente en las áreas científicas necesarias para la innovación tecnológica. Esto último requiere una inversión en los niveles educativos superiores que formen a personas capaces de crear conocimiento (Chen y Dahlman 2004). En este sentido, este conocimiento adquirido servirá de base para crear nuevas empresas fomentando el autoempleo e influyendo en la tasa de desempleo de un país.

Es fundamental para los países conocer los factores en los cuales deben de influir si desean mejorar a través del conocimiento, pero también lo es conocer su situación actual. Para dar respuesta a esto, se han comparado las principales variables de inversión y resultado de las distintas organizaciones analizadas: gasto público en $\mathrm{I}+\mathrm{D}$ (ver Figura 1), gasto público en tecnologías de la información y la comunicación (TIC) (ver Figura 1), gasto público en educación (ver Figura 2), número de patentes por millón de habitantes (ver Figura 3), número de artículos científicos por millón de habitantes (ver Figura 3) y ratio de nuevas empresas (ver Figura 4). 
Figura 1. Comparación entre los grupos de países de las inversiones realizadas en $\mathrm{I}+\mathrm{D}$ y TIC

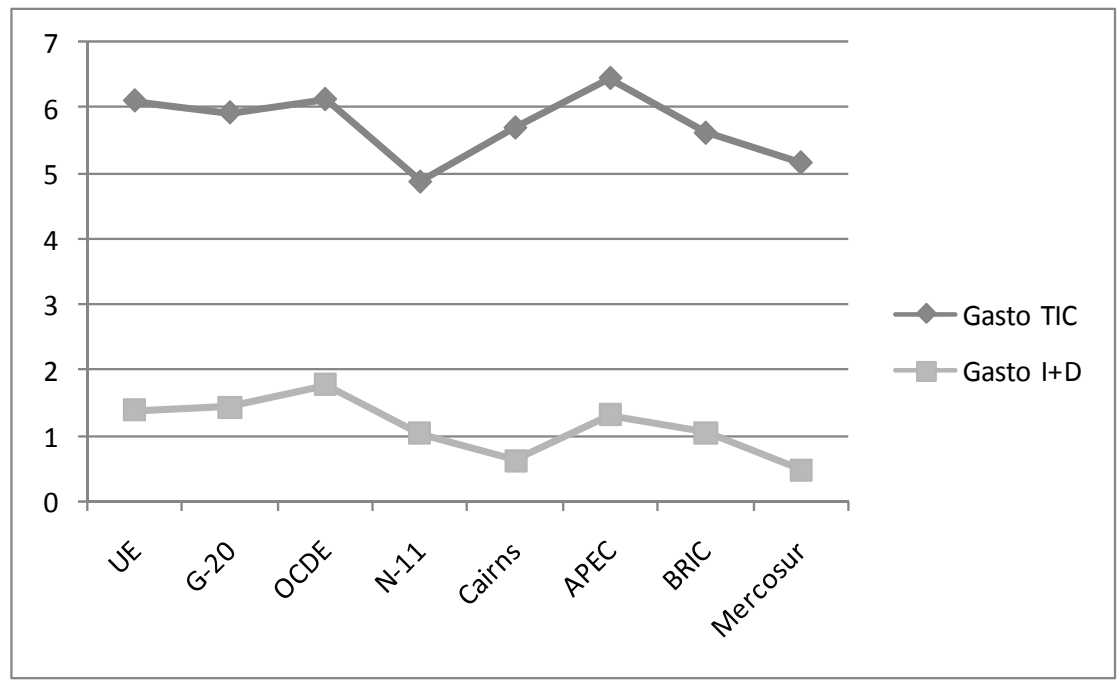

Fuente: Elaboración propia

La mayor inversión en investigación y desarrollo fue realizada por la OCDE (1,77 por ciento sobre el PIB), seguida de cerca por los países que forman parte del G-20 (1,43 por ciento), la Unión Europea (1,38 por ciento) y los APEC (1,32 por ciento). A mayor distancia se sitúan los países BRIC (1,04 por ciento) y los N-11 (1,03 por ciento). Finalmente, a una distancia considerable respecto a los primeros, se sitúan los países Cairns, con una inversión de 0,61 por ciento sobre el PIB y aquellos que conforman el MERCOSUR, con una inversión en I+D de 0,48 por ciento sobre el PIB (ver Figura 1).

En cuanto a la inversión en TIC son los países APEC, con un 6,44 por ciento sobre el total de gasto del gobierno, los que lideran la lista. En segundo lugar se sitúan los países de la OCDE (6,12 por ciento) y aquellos que forman parte de la Unión Europea (6,10 por ciento). Una inversión cercana a estos agrupaciones realizan el G-20 (5,92 por ciento), los países Cairns (5,69 por ciento) y los BRIC (5,61 por ciento). Las inversiones más bajas las realizan los países MERCOSUR ( 5,16 por ciento) y los $\mathrm{N}-11$ ( 4,87 por ciento), con una diferencia de más de un punto porcentual con respecto a los APEC (ver Figura 1).

En contraposición con la inversión en TIC, donde estaban en última posición, los países conocidos como N-11 lideran la inversión en educación con un 17,36 por ciento con respecto al gasto total del gobierno, seguidos muy de cerca por los APEC (17,18 por ciento). La tercera posición la ocupan los países Cairns, con una inversión en educación del 16,09 por ciento con respecto al total de gasto del gobierno. Los países BRIC (15,62 por ciento), los MERCOSUR (15,38 por ciento) 
y los que forman parte del G-20 (15,34 por ciento) realizarían inversiones situadas en posiciones intermedias. Finalmente los países de la OCDE, con una inversión de 13,79 por ciento con respecto al total de gasto del gobierno, y aquellos que forman parte de la Unión Europea, que invirtieron de media un 12,04 por ciento, se sitúan en las últimas posiciones (ver Figura 2).

Figura 2. Comparación entre los de países de la inversión realizada en educación

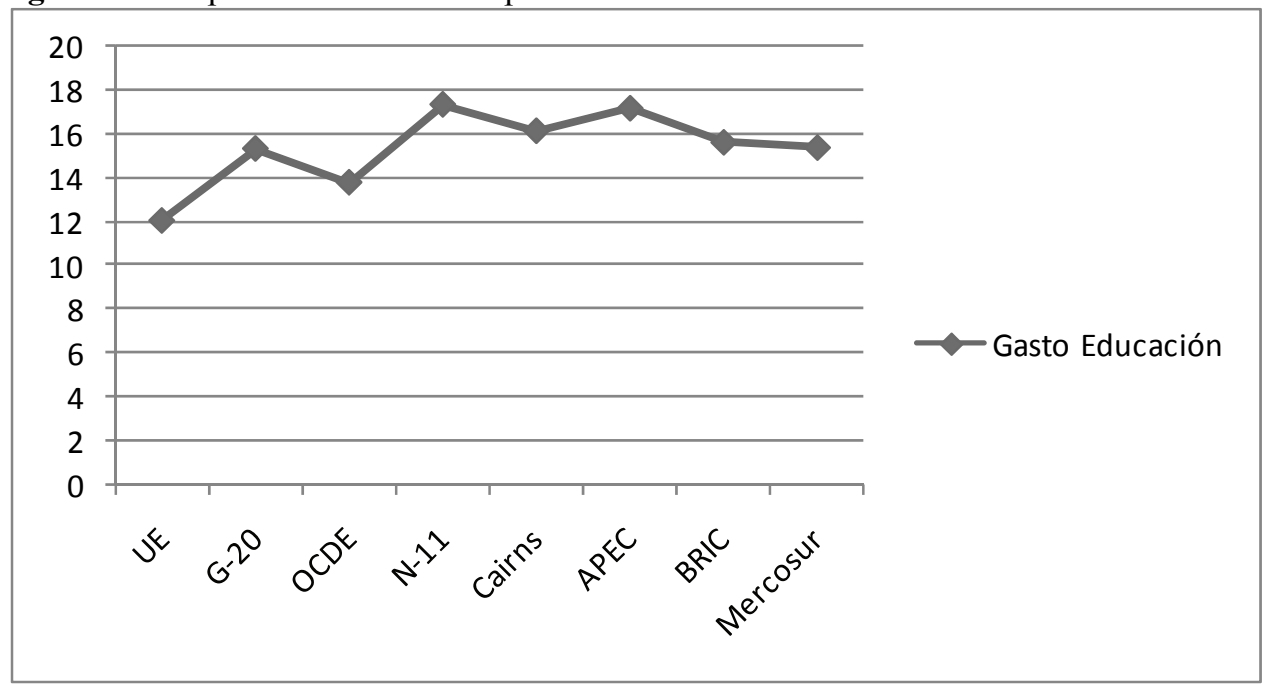

Fuente: Elaboración propia

En cuanto a los resultados, el mayor número de patentes lo consiguen los países que conforman el G-20 (421 patentes por millón de habitantes), seguido muy de cerca por los APEC, quienes alcanzaron 398 patentes por millón de habitantes y los que forman parte de la OCDE (325 por millón de habitantes). Los países conocidos como N-11 alcanzaron las 265 patentes por millón de habitantes, mientras que la Unión Europea ofrece una media de 137. En las últimas posiciones se encuentran los BRIC (65 patentes por millón), los Cairns (48 patentes por millón de habitantes) y los países MERCOSUR (14 patentes por millón) (ver Figura 3).

El número de artículos científicos también era considerado como una variable de resultado relacionada con el conocimiento. En este caso los países de la OCDE ocupan el primer puesto con 538 artículos científicos por millón de habitantes, seguidos por la Unión Europea, con una media de 414 artículos. A una distancia considerable se sitúan los APEC y los que forman el G-20 (301 artículos por millón de habitantes cada uno). A una gran distancia se sitúan los Cairns (148 artículos por millón de habitantes) y finalmente las últimas posiciones son para los MERCOSUR, los N-11 y los BRIC, con 59, 50 y 49 artículos científicos por millón de habitantes respectivamente (ver Figura 3). 
Figura 3. Comparación entre grupos de países del número de patentes y el número de artículos obtenidos por millón de habitantes

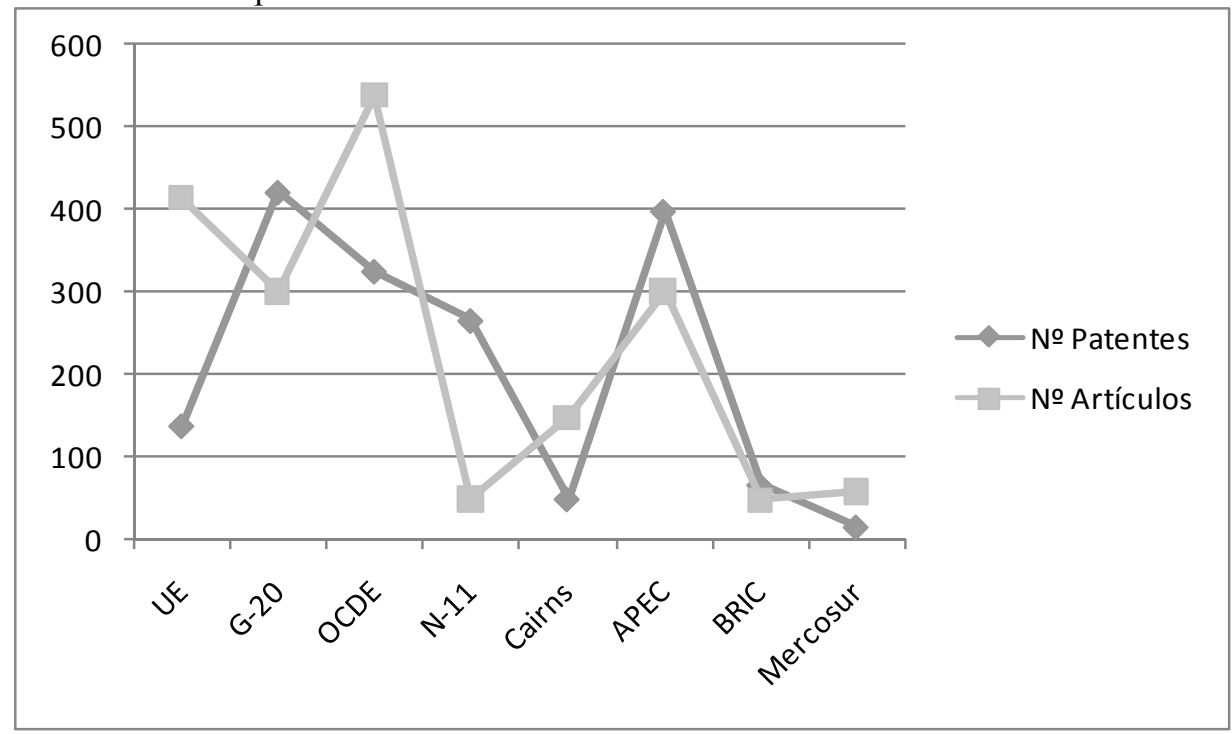

Fuente: Elaboración propia

La última variable seleccionada hace referencia al ratio de nuevas empresas. En este caso las primeras posiciones las ocupan los países APEC (3486 nuevas empresas por millón de habitantes), la Unión Europea (con una media de 3400 nuevas empresas por millón de habitantes) y los países de la OCDE (3163 nuevas empresas por millón de habitantes). El grupo G-20 y los países Cairns siguen la lista con 2043 y 2018 nuevas empresas por millón de habitantes respectivamente. En último lugar se sitúan los BRIC (1790 nuevas empresas por millón de habitantes), los MERCOSUR (1406 nuevas empresas por millón de habitantes) y los N-11 (980 nuevas empresas por millón de habitantes).

Los países pertenecientes a la OCDE, el G-20 y la Unión Europea, que coinciden con los países más poderosos del mundo, realizan fuertes inversiones en I+D y TIC, mientras que sucede lo contrario con las inversiones en educación. Esto puede ser debido precisamente a que la infraestructura y los niveles de educación de estos países son más satisfactorios $\mathrm{y}$, por tanto, necesitan una menor inversión. En relación a las variables de resultado y con carácter general, estas tres agrupaciones de países consiguen unos resultados excelentes, destacando especialmente la posición en estos de los países de la OCDE. Esto tiene sentido, puesto que la OCDE incluye las 34 naciones más ricas del mundo y también es conocida como club de los países ricos. 
Figura 4. Comparación entre las distintas agrupaciones de países del ratio de nuevas empresas.

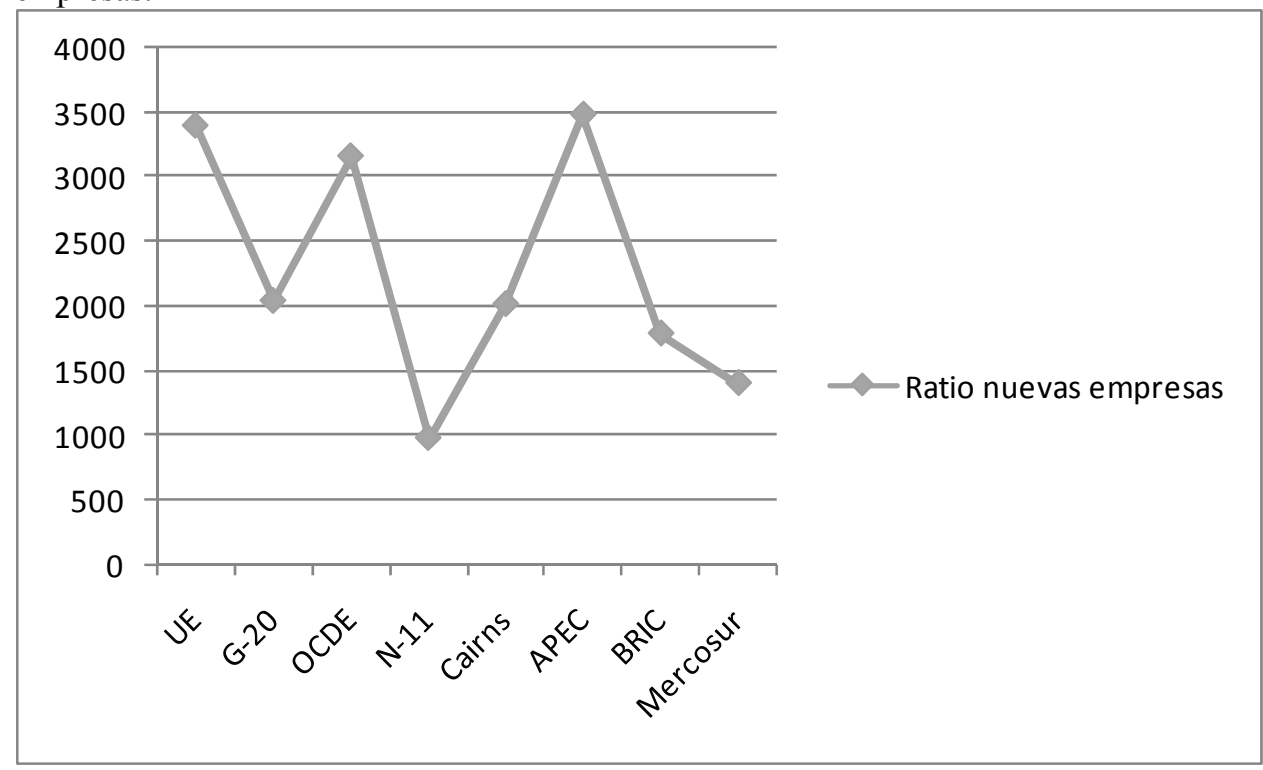

Fuente: Elaboración propia

Los países APEC realizan inversiones medias en I+D y educación, mientras que en el caso de las TIC su inversión es alta. Los resultados obtenidos por los países APEC son medios-altos, lo que es congruente con las inversiones realizadas.

Por su parte las agrupaciones de países BRIC y NEXT-11, realizan inversiones medias en I+D, TIC y educación, destacando especialmente la inversión que realizan los NEXT-11 en educación. Los países que conforman la agrupación NEXT-11 son países con menor grado de desarrollo cuyo despegue está comenzando ahora, lo que está en línea con una mayor inversión en este punto. En cuanto a los resultados, en ambos casos son bajos. Esto coincide con las inversiones que realizan.

Finalmente los países que conforman MERCOSUR y Cairns se caracterizan por ser los que menos invierten y también los que obtienen los peores resultados.

\section{CONCLUSIONES}

Utilizando la técnica del análisis factorial se han identificado cinco parámetros de éxito organizacionales que deben ser tenidos en cuenta por los gobiernos interesados en promover el crecimiento de su economía, fundamentado en una eficiente estructura del conocimiento. El primer factor, que se destaca como el 
fundamental debido a que representa el 29 por ciento de la variabilidad, se denomina Potencial de creación de conocimiento, y recoge, entre otras, variables necesarias para la creación de conocimiento en los países como la inversión en I+D o los trabajadores del conocimiento. El segundo de los factores, Productividad TIC recoge variables relacionadas con la tecnología de la información y la comunicación. El tercer factor ha sido denominado Internacionalización del conocimiento y está relacionado as con el intercambio de conocimiento entre países. Los factores 4 y 5 son los menos representativos a la hora de gestionar conocimiento en los países. El factor 4, Resultados de investigación recoge la importancia de los resultados a la hora de diferenciar entre países; por otro lado el quinto y último factor, Autopista de la educación, destaca la importancia de la inversión en educación para el crecimiento de un país y representa el 7 por ciento de la variabilidad.

En relación con el análisis de las diferencias entre las agrupaciones, se puede establecer que aquellas cuya inversión media es superior coinciden, en general, con los mejores resultados. En este grupo se encuentran: OCDE, G-20, Unión Europea y APEC. Por otro lado, aquellas cuya inversión media es más baja coinciden, en general, con los peores resultados. En este grupo se sitúan: MERCOSUR, Cairns, BRIC y N-11.

En la sociedad actual donde la gestión del conocimiento tiene una presencia clara, las contribuciones originales de esta investigación tienen varias implicaciones prácticas. En primer lugar, para los gobiernos de los países analizados, ya que los parámetros de éxito identificados suponen una ayuda en la toma de decisiones permitiéndoles centrar esfuerzos en los aspectos más fundamentales. Por otro lado, el análisis de las diferentes agrupaciones les permite conocer su situación y, si es necesario, incrementar el esfuerzo en conocimiento. De esta forma, países que forman parte de la OCDE, G-20, Unión Europea y APEC deben de mantener sus estrategias de conocimiento, mientras que países que forman parte de MERCOSUR, Cairns, BRIC y N-11 deberían de redoblar sus esfuerzos en esta materia.

En relación con el área académica que investiga la gestión del conocimiento en las organizaciones, este análisis contribuye a un hueco existente en el área que ahora está comenzando a recibir más atención: la gestión del conocimiento en países. En este sentido supone un nuevo enfoque y, por lo tanto, un punto de partida para futuras investigaciones.

Las limitaciones que presenta la investigación son las siguientes: (1) aquellas propias de la adquisición de los datos por parte del Banco Mundial, (2) el año de análisis seleccionado y (3) la muestra de países analizados, puesto que debido a falta de información solo se han seleccionado 67 países. 


\section{BIBLIOGRAFÍA}

ADAMS, James D. 1990. Fundamental stocks of knowledge and productivity growth. Journal of Political Economy, Vol. 98, no. 4. 1990. p. 673-702.

BANCO MUNDIAL DATABASE. 2010. Página web oficial: http://www.bancomundial.org.

BRESHANAN, Timothy F., BRYNJOLFSSON, Erik y HITT, Lorin M. Information technology, workplace organization and the demand for skilled labor: Firm-level evidence. Quarterly Journal of Economics, Vol. 117, no. 1. 2002. p. 339-376.

CASTIGLIONI, Concetta. 2009. ICT investment and firm technical efficiency. Paper presented at XI European Workshop on Efficiency and Productivity Analysis (EWEPA), Pisa, Italia.

CERQUERA, Daniel y KLEIN, Gordon J. Endogenous firm heterogeneity, ICT and R\&D incentives. ZEW Discussion Paper No. 08-126. 2008.

CHEN, Derek H.C. y Dahlman, Carl J. Knowledge and development: A crosssection approach. World Bank Policy Research, Working paper \#3366. 2004.

DRUCKER, Peter. The new productivity challenge. Harvard Business Review, sept-oct. 1991. 95-104.

DRUCKER, Peter. The new society: the anatomy of industrial order. Nueva York: Harper \& Row, 1993. ISBN: 978-1560006244.

FLETCHER, Louise y POLYCHRONAKIKS, Yiannis E. Capturing knowledge management in the supply chain. EuroMed Journal of Business, Vol. 2 no. 2. 2007. p. 191-2007.

GANS, Joshua S., MURRAY, Fiona E. y STERN, Scott. Patents, papers, pairs \& secrets: Contracting over the disclosure of scientific knowledge. Working paper, 2005.

GREENAN, Nathalie, TOPIOL-BENSAID, Agnes, y MAIRESSE, Jacques. Information technology and research and development impacts on productivity and skills: Looking for correlations on French firm level data. En Information Technology, Productivity and Economic Growth. Oxford: Oxford University Press, Pohjola, M. (ed.), 2001.

GUELLEC, Dominique, y VAN POTTELSBERGHE DE LA POTTERIE, Bruno. R\&D and productivity growth: Panel data analysis of 16 OECD Countries. Organisation for Economic Co-operation and Development, STI Working Papers, 2001.

HAIR, Joseph F., BLACK, Bill, BABIN, Barry, ANDERSON, Rolph, E. y TATHAM, Ronald, L. Multivariate data analysis. Englewood Cliffs, NJ: Prentice Hall, 1995.

JIMES, Cynthia, y LUCARDIE, Larry. Reconsidering the tacit-explicit distinction - A move toward functional (tacit) knowledge management. Electronic Journal of Knowledge Management, Vol. 1, no. 1. 2003. p. 23-32. 
JONES, Charles I. Sources of US economic growth in a world of ideas. The American Economic Review, Vol. 92 no. 1. 2002. p. 220-39.

KAKABADSE, Nada K., KOUZMIN, Alexander y KAKABADSE, Andrew. From tacit knowledge to knowledge management: Leveraging invisible assets. Knowledge and Process Management, Vol. 8 no. 3. 2001. p. 137-154.

KEBEDE, Gashaw. Knowledge management: An information management perspective. International Journal of Information Management, 30. 2010. p. 416-424.

KIM, J-O. y MUELLER, C.W. Factor analysis: statistical methods and practical issues. Part II. En Factor analysis and related techniques. Londres: Sage, M.S. Lewis-Beck (ed.), 1994.

LEDERMAN, Daniel y MALONEY, William F. R\&D and development. The World Bank, Policy Research Working Paper 3024, 2003.

MACINTOSH, Ann. Position paper on knowledge asset management. Artificial Intelligence Applications Institute, University of Edimburgh, Escocia. 1996.

MARTINUS, Kirsten. Planning for production efficiency in knowledge-based development. Journal of Knowledge Management, Vol. 14, no. 5. 2010. p. 726743.

MAC MORROW, Noreen. Knowledge management: an introduction. Annual Review of Information Science and Technology, Vol. 35. 2001. p. 381-422.

NUSSBAUM, Martha. Women and human development: The capabilities approach. Cambridge, RU: Cambridge University Press, 2000. ISBN: 9780521003858 .

OBESO, Maria, SARABIA, Maria y SARABIA, Jose M. Gestionando conocimiento en las organizaciones: Una visión actual de la gestión del conocimiento. Alemania: Editorial Academica Española, 2012. ISBN: 9783659038303.

OLUIC-VUKIVIC, Vesna. From information to knowledge: Some reflections on the origin of the current shifting towards knowledge processing and further perspective. Journal of the American Society for Information Science and Technology, Vol. 52, no. 1. 2001. p. 54-61.

PASSERINI, Katia. Knowledge-driven development indicators: Still an eclectic panorama. Journal of Knowledge Management, Vol. 7, no. 11. 2007. p. 115128.

POLDER, Michael, VAN LEEUWEN, George, MOHNEN, Pierre y RAYMOND, Wladimir. Productivity effects of innovation modes. Statistics Netherlands Discussion, Paper no 09033, 2009.

POOLE, Erik y BERNARD, Jean Thomas. Defence innovation stock and total factor productivity growth. Canadian Journal of Economics, Vol. 25, no. 2. 1992. p. 438-52.

ROMER, Paul M. Endogenous technological change. Journal of Political Economy, Vol. 98, no. 5. 1990. p. 71-102. 
SAKAIYA, Taichi. The knowledge value revolution or a history of the future. Tokio: Kodansha International, 1991. ISBN.

SARABIA, Maria, OBESO, Maria y SARABIA, Jose M. How do European countries manage their knowledge? A cross analysis of investment and performance. Innovation: Management, Policy \& Practice, 14(1). 2012. p. 129142.

SHARRATT, Mark y USORO, Abel. Understanding knowledge-sharing in online communities of practice. Electronic Journal on Knowledge Management, Vol. 1, no. 2. 2003. p. 187-196.

VILLELA DANTAS, José R. y MUNIZ FARIAS, Pedro P. 2010. Conceptual navigation in knowledge management environments using NavCon. Information Processing and Management, Vol. 46. 2010. p. 413-425.

ZHOU, Shihao, SIU, Felix y WANG, Minhong. Effects of social tie content on knowledge transfer. Journal of Knowledge Management, Vol. 14, no. 3. 2010. p. 449-463. 